selves vary in shape sinusoidally. Fig. 1 shows a unit of area used to build up the master figure. Each black streak in Fig. 1 obeys the equation

$$
\omega=\omega_{0}\left(1+\sin \frac{2 \pi n x}{L}\right) \text {, }
$$

where $\omega$ is the width at any distance, $x$, from the left-hand edge, $\omega_{0}$ is the maximum width, and $L$ is the length of the streak.

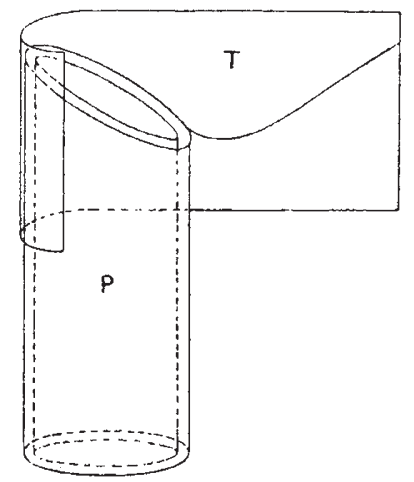

Fig. 2. Manner of making a template for drawing sine curves

Since it is very difficult to plot, from trigonometrical tables, these sinusoidal curves sufficiently accurately for this work, a template was made by the procedure shown in Fig. 2. A piece of 5 .mill shim brass ' $T$ ' is wrapped about a pipe $P$ with a bevelled end. The inside of the shim brass is marked against the bevelled end of the pipe with a sharp metal scribe. When the brass is unwrapped from the pipe, a sine curve is apparent; and by cutting the brass on the scribed line, the template is ready for making curves and filling in with ink to produce Fig. 1.

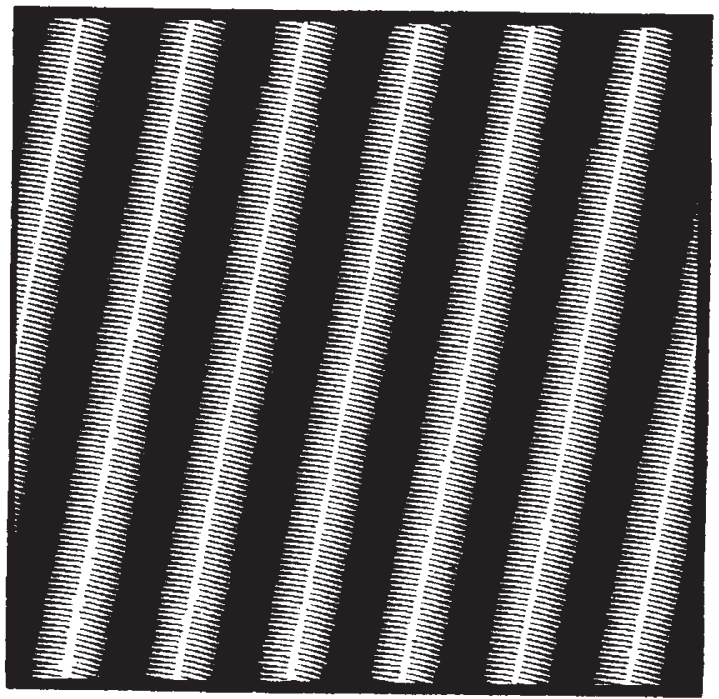

Eig. 3. Example of flnished mask, showing the 61 term

Fig. 3 shows the $6 \overline{\mathbf{l}}$ mask. Masks up to $h$ and $k$ values of 10 have been prepared and are available through the Universal Microfilming Corporation (277 6th Avenue, Salt Lake City 3, Utah). When these masks are used in the usual manner or in the multiple projector ${ }^{4}$, the details of the white and black streaks are lost and a sinusoidal effect of dark and light bands is produced by out-of-focus projection.

${ }^{1}$ Bragg, W. L., Z. Krist., A, 70, 475 (1929).

${ }^{2}$ Huggins, M. I., J. Amer. Chem. Soc., 63, 66 (1941).

${ }^{3}$ Huggins, M. L., J. Chem. Phys., 12, 520 (1944).

"Woolley, R. H., and McLachlan, jun., D., a paper before the American Society for X-Ray and Electron Difl raction. "A Multiple Pro-
jector for the Huggins Masks" (Ithaca, New York, June 1949).

\section{APPLIED ELECTRONICS}

7 HE exhibition of electronic devices which was held at the Manchester College of Technology during the last week of July was the sixth, and by far the largest, of the annual displays organized by the North-West Branch of the Institution of Electronics. The Exhibition, planned as part of the City's contribution to the Festival of Britain activities, included a number of special features in addition to the usual trade displays of production models of electronic equipment. The research section, in which applications of electronics were on show in the 'string and sealing-wax' stage, was particularly interesting; and there were a number of 'guest' exhibits which, in addition to being excellent examples of applied electronics, were also of high entertainment value. Film shows and a series of lectures on specific problems were also part of the programme.

In the space allowed, it would be impossible to describe all the exhibits which were of special interest, and it must be pointed out that the particular devices selected for mention here are not necessarily of greater merit than the rest but are simply those which had the greatest personal appeal to the writer.

Of the 'guest' exhibits, perhaps the most impressive was the 'Electrone' displayed by the John Compton Organ Co., Ltd. This attracted a lot of attention as it was a smaller version of the instrument to be installed in the Manchester Free Trade Hall. The console of the instrument is completely orthodox; the stops, pistons and manuals are identical with those to be found on a modern pipe organ, and in fact no additional knowledge or skill is required by the player than would be required for playing an equivalent pipe organ. The tones are generated electrostatically from capacity changes produced by rotating wave-form electrodes. Harmonics are not produced separately but in groups, so that wide varieties of tone can be produced with a minimum of circuits. Valves are used only for straight amplification. Although the organ occupies a very small amount of floor space, it has a tonal quality which is remarkably close to that of a good pipe organ. Specially designed circuits provide delays (both before and after the depression of a key) which simulate the speaking characteristics of actual pipes with remarkable accuracy, and the instrument has none of the characteristics usually associated with electronic musical instruments.

Ferranti, Ltd. (Edinburgh), displayed a slow-speed relay computer designed to demonstrate the technique of automatic solution of problems in logic. Complex logical problems can, when broken down into a number of 'rules' or logical restrictions, be solved in a very short time even by this slow-speed machine. Two models were demonstrated. One obtained the solution by trying all possible combinations of the variables in turn; this model would give all the possible solutions to a given problem. The other does not try all combinations but is made to enverge 
on a correct solution; this form is much more rapid, can deal with a greater number of variables but gives only one of the possible answers.

Also among the 'guest' exhibits was an amateur radio station in full operation, complete with homemade radio transmitters and receivers and a television receiver.

In the research section, Imperial Chemical Industries (Dyestuffs Division), Ltd., displayed a light-flux integrator. A photo-cell charges a condenser which triggers a flip-flop circuit at a given charge-level. This operates a counter, discharges the condenser and resets the circuit for the next count. A range of instruments involving transformer-type electromagnetic displacement gauges were on show. One such device measured the rate of wetting of a textile sample in water by recording the movements of a torsion balance, to which the sample was attached, as the water was absorbed.

The Electrical Engineering Department of the Manchester College of Technology displayed apparatus illustrating both research carried out in the College, and devices used in teaching. In the former category was an apparatus for indicating the rate of change of mains-frequency directly on a single dial, and designed for use in power stations. A useful device for teaching purposes was a harmonic synthesizer in which one could see, directly on a cathoderay tube screen, the effect of adding together various proportions of different harmonics.

In the trade section there was a bewildering variety of equipments, all beautifully finished and a large proportion of them actually in operation.

Ferranti, Ltd. (Hollinwood), demonstrated their mercury-argon crater lamps, which give a light output directly proportional to the current passing through the lamp. These have wide application in sound-film recording and picture transmission, as the light can be modulated at frequencies up to $12,000 \mathrm{c} . / \mathrm{s}$. Among other interesting items was a new flat-faced television picture-tube with a 14-in. diagonal rectangular screen.

Fielden (Electronics), Ltd., demonstrated a yarnirregularity meter. The instrument effectively determines the mass of a centimetre length of yarn, instantaneously present in the gap of a special condenser head, in terms of its dielectric properties. The instrument then notes the irregularities, integrates them over a predetermined period and presents the result, on a single dial, as the average irregularity expressed as a percentage of the average mass of the sample. The reading is quite steady and independent of the speed at which the sample is passing through the instrument.

One of the most interesting exhibits on the stand of Marconi Instruments, Ltd., was an audiometer assembly, specially designed for measuring the hearing acuity of young children. The instrument incorporates a standard pure-tone audiometer. The portion of the instrument visible to the child is brightly decorated in nursery style and consists of a loud-speaker and a 'peep-show' arrangement. This is so devised that a brightly coloured picture becomes visible when the child presses a button, provided that a test signal is being applied to the loud-speaker.

The Metropolitan-Vickers Electrical Co., Ltd., displayed their high-speed, recurrent-wave-form monitor which gives, on a cathode-ray tube screen, a trace of a recurrent wave-form with frequency components up to $300 \mathrm{Mc} / \mathrm{s}$. In any one cycle, the instantaneous amplitude at some one point of the wave-form is measured. On successive recurrences, the instantaneous value at other points is recorded and after about 200 cycles a complete picture of the wave is available.

Mullard, Ltd., had on view a number of important additions to their range of valves, including a new disk-seal ultra-high-frequency triode, with a new type of cathode which permits closer spacing of electrodes -with consequent higher maximum frequency of operation - than has hitherto been obtainable, a səries of sub-miniature valves and the first members of their range of image-converter tubes.

The Exhibition Committee deserves special congratulations on the organization, which, at any rate to an outside observer, appeared to be particularly free from technical hitches. $\quad$ C. A. TAYLor

\section{RESEARCH AND DEVELOPMENT IN THE SIMON ENGINEERING GROUP}

\author{
By DR. W. RHEINALLT THOMAS \\ Director of Research
}

$T$

HE year 1908 saw the establishment of the first Simon Laboratory for examining and testing the materials used and produced in the coke ovens and coal-washing plant then being built by the firm. In 1912 tests associated with the manufacture of sulphuric acid were also undertaken. In the period between the two World Wars, the Laboratory was enlarged on three occasions to cater for advances in the design of existing plant and the introduction of new activities, which now include the production of power stations, turbine gears and a wide variety of chemical plant and mechanical and pneumatic handling machinery. Each department had also set up its own small teams of research workers and technical development engineers.

Tremendous expansion followed the Second World War, as demonstrated by the fact that orders to the value of $£ 75$ million have been taken at home and abroad, and it became clear that existing arrangements for testing, research and development were inadequate. Plans were drawn up to meet the situation, and building began in mid-1949.

On July 3 last, Sir Henry Tizard opened the new buildings, consisting of a central research block, a testing wing housing what was known as the Laboratory and now forming part of the Research Department, and the first of two development wings. These new buildings are almost entirely devoted to the research and development interests of SimonCarves, Ltd. The Henry Simon companies forming the other half of the Simon Engineering Group, and with very different fields of activity, have entirely independent facilities for research and development, although there is the closest co-operation, particularly where problems of mutual interest are concerned.

The central research building, $159 \mathrm{ft}$. long, $45 \mathrm{ft}$. wide and $45 \mathrm{ft}$. high, contains four research laboratories, microscope, balance and dark rooms, as well as sections for testing metals and refractory and building materials. The library, information room and offices occupy the central portion of the first floor.

The testing wing on two floors is $105 \mathrm{ft}$. Iong, $55 \mathrm{ft}$. wide and $25 \mathrm{ft}$. high, and is specially designed to cater, on a much enlarged scale, for the activities of 